\title{
Incidental Papillary Endothelial Hyperplasia of the Cavernous Sinus: Case Report and Review of the Literature
}

\author{
*Peter S. Amenta \\ Department of Neurosurgery, University of \\ Miami, 1095 NW $14^{\text {th }}$ Terrace \\ Miami, FL, USA \\ peter.amenta@gmail.com \\ Jose E. Velazquez-Vega \\ Department of Pathology, University of Miami, \\ 1095 NW $14^{\text {th }}$ Terrace \\ Miami, FL, USA \\ jose.velazquezvega@jhsmiami.org
}

\author{
Andrew E. Rosenberg \\ Department of Pathology, University of \\ Miami, 1095 NW $14^{\text {th }}$ Terrace \\ Miami, FL, USA \\ ARosenberg@med.miami.edu \\ Jacques J. Morcos \\ Department of Neurosurgery, University of \\ Miami, 1095 NW $14^{\text {th }}$ Terrace \\ Miami, FL, USA \\ JMorcos@med.miami.edu
}

\begin{abstract}
:
Background and Importance: Intravascular papillary endothelial hyperplasia (IPEH), a pathologic variant of thrombus organization, is driven by endothelial cell proliferation along surfaces of aggregate fibrin, resulting in the formation of papillary structures. Intracranial IPEH is very rare, and is infrequently encountered at the cranial base. Progressive lesional growth results in mass effect on adjacent structures leading to various neurologic deficits.

Clinical Presentation: A 40 year-old woman presented with symptoms of hyperprolactinemia. MRI revealed a microadenoma and a large left-sided asymptomatic lesion that filled the cavernous sinus. Following the growth of the lesions on imaging, both were resected via the endoscopic endonasal route. The cavernous sinus mass was subtotally resected, as it was densely adherent to the critical surrounding neurovascular structures. Histopathologic and immunohistochemical examination confirmed the diagnosis of IPEH. At six-month followup, MRI revealed a large recurrence of the IPEH, which remained asymptomatic.

Conclusion: IPEH is a rarely encountered intracranial pathology that presents significant treatment challenges. Curative treatment is through complete surgical resection, however, in skull bases lesions, this goal may be difficult to achieve without significant morbidity. Incompletely resected IPEH can recur, sometimes rapidly and aggressively, and a treatment algorithm for such cases has not been established.
\end{abstract}

Keywords: Intravascular papillary endothelial hyperplasia;cavernous sinus;skull base lesions;endoscopic resection

\section{INTRODUCTION}

Intravascular papillary endothelial hyperplasia (IPEH) was first described by Pierre Masson in 1923 in a thrombosed hemorrhoidal vein.[17] Early investigations questioned the origin of these lesions and consideration was given as to whether IPEH represented a sarcoma. IPEH is not considered to be a neoplastic process, and is considered to be a manifestation of a pathologic variant of thrombus organization formed byproliferating endothelial cells that produce papillary structures as they grow along the surfaces of aggregates of fibrin.[2, 5, 6, 14]IPEH occurs in different settings and has a female predisposition (M:F; 7:1) and often develops in thesuperficial soft tissues of the face and fingers.[7, 21] Intracranial IPEH israre, and may occur as an extra-axial or intraparenchymal mass.[8, 15] Progressive lesional growth results in mass effect on adjacent structures leading to various neurologic deficits. Curative treatment is through complete surgical resection, however, in skull bases lesions, this goal may be difficult to achieve without significant morbidity. Incompletely resected IPEH can recur, sometimes rapidly and aggressively, and a treatment algorithm for such cases has not been established. We present a case of a young woman who presented to our clinic with a pituitary adenoma and a large left-sided cavernous sinus lesion found to be IPEH upon histological examination. 


\section{CASE REPORT}

A 40 year-old female presented with a two-year history of amenorrhea.Work-up revealed a high serum prolactin and magnetic resonance imaging (MRI) demonstrated a $6 \mathrm{~mm}$ left-sided pituitary adenoma confined to the sella. Additionally, an approximately $2 \mathrm{~cm}$ homogenously enhancing left cavernous sinus mass extending into the sphenoid sinus was also incidentally discovered. This second lesion was felt to be most-likely a trigmeninalschwannoma, however, cavernous hemangioma and capillary angioma remained in the differential. The patient was neurologically intact on examination and was counseled on close observation with serial imaging of the asymptomatic cavernous sinus mass. A regimen of cabergoline was initiated for the prolactinoma and the symptoms resolved. On follow-up MRI at eight months, the lesions demonstrated growthand the decision was made to address both with surgical resection simultaneously through an endoscopic endonasal route.

The symptomatic pituitary lesion was resected first without incident and histologic examination confirmed apituitary adenoma.Immunohistochemical staining confirmed the diagnosis of prolactinoma. Upon entering the cavernous sinus, a reddish encapsulated mass that appeared highly vascular was encountered. Due to the size of the lesion, circumferential dissection proved difficult and internal debulking was needed to collapse the mass inward. Opening of the capsule resulted in profuse venous bleeding that was difficult to control. Packing of the region with powdered GELFOAM (Pfizer), Surgicel (Ethicon, Inc.), and cottonoids slowed the bleeding and permitted significant debulking of the mass, however, complete resection was not possible due to the lack of a plane with the structures of the cavernous sinus. Histopathological examination of the lesion revealed IPEH. Postoperative MRI demonstrated complete resection of the pituitary adenoma and a small residual component of the IPEH. The patient was discharged neurologically intact.

The patient returned for her six-month follow-up reporting a normal menstrual cycle and without any new signs or symptoms. She remained neurologically intact on examination. MRI performed at this time revealed a significant recurrence of the IPEH within the left cavernous sinus. The patient underwent Gamma knife radiation for the recurrence and will be followed closely with serial MRI.

\section{DISCUSSION}

IPEH accounts for approximately $2 \%$ of subcutaneous vascular lesions of the head and neck and extremities, particularly the hands, and may also be found in the lips, tongue, and buccal mucosa.[16, 18, 19, 25] Rarely, IPEH has been discovered in muscles, the digestive and urogenital tracts, the upper respiratory tract mucosa, the heart and other internal organs. $[9,11,14]$ The soft tissue masses are observed to have a bluish or reddish discoloration and may be tender to palpation.[14]While some studies do not report a sex predilection, others have found that females, predominantly in their forties, are more commonly affected than men.[21]Three settings of the disease have been described as follows:Type I (primary or pure) IPEH develops within a histologically normal yet dilated vessel, which is usually a vein, although arteries may rarely be involved. Type II (mixed IPEH) occurs in preexisting vascular lesions, such as, hemangiomas, venous lakes, thrombosed aneurysms, arteriovenous malformations, pyogenic granulomas, and lymphangiomas. Type III arisesin the setting of a hematoma. All three types are linked by an underlying disturbance of blood flow and thrombosis from which the mass lesion evolves.[11, 13, 19]

IPEH is a pathologic variant of thrombus organization and revascularization. Once formed, thrombus can follow four distinct pathways, including propagation, embolization, fibrinolytic dissolution, and organization and recanalization.[13] As thrombi organize, fibrin polymerization renders the clot less vulnerable to dissolution. Ingrowth of endothelial cells and fibroblasts can result in neovascularization and capillary proliferation. Anastomoses between these vessels may re-establish blood flow through the organizing mass of connective tissue, resulting in a highly vascular lesion. [8, $10,15,16,19]$

Histologically, IPEH is heterogeneous in appearance consisting of multiple areas in various stages of thrombus organization and revascularization.[13]In the early stage, the lesion consist of fibrin clot which serves as a scaffolding for proliferating endothelial cells that dissect into the thrombus. These cells formpapillae composed of a central core of fibrin lined by plump metabolically active endothelial cells expressing the immunohistochemical markers Factor VIII-related antigen, CD31, CD34, and ERG.[13, 18]The thrombus is chemotactic for macrophages that help remove the fibrin, which is eventually replaced by collagen. This process results innewly formedinterconnecting spaces 
thatcreate a complex vascular network. The combination of the cytologic features of the endothelial cells and the architectural pattern is a known diagnostic pitfall and can be confused with angiosarcoma. However, features of malignancy, including, abundant mitoses with atypical forms, invasion of adjacent structures, necrosis, and severe nuclear pleomorphism, are absent.

The pathophysiology of IPEH includes activation of different cytokines,such as, fibroblast growth factor protein, vascular endothelial growth factor (VEGF), epidermal growth factor (EGF), plateletderived growth factor (PDGF), and hypoxia-inducible-factor-1 (HIF-1). ${ }^{16},[1,4,12]$. The molecules recruit and activate the involved cell populations, including, endothelial cells, macrophages, and fibroblasts.

Intracranial IPEH is exceedingly rare and has been reported to involve the parenchyma, meninges, venous sinuses, superior orbital fissure, and the skull base.[15, 19, 22, 23] There has been some debate as to whether the true incidence of intracranial IPEH has been underreported in the literature.[14] Due to the relative rarity of the disease, IPEH is often misdiagnosed on CT and MRI as a meningioma,schwannoma, angiosarcoma, chordoma, chondrosarcoma, or inflammatory lesion. CT usually demonstrates a circumscribed hyperdense mass.[8, 10] When found in the skull base, significant bone erosion may be observed. IPEH appears as a highly vascular isointense or mildly hyperintense mass on T1-weighted MRI and hyperintense on T2 sequences.T1-weighted MRIwith gadolinium reveals an intensely enhancing homogenous mass.[8, 21] Angiographic findings are variable and range from angiographically occult to a vascular blush.[18, 23]

The clinical presentation of IPEH is variable and dependent upon the location of the lesion. Due to the slow growing nature of the lesion, IPEH may achieve a large size prior to becoming symptomatic. Lesions in the cavernous sinus, superior orbital fissure, and orbit result in ophthalmoplegia, proptosis, retro-orbital pain, and visual loss. Intralesional hemorrhage may result in rapid growth and acute neurologic deficits. The natural history of intracranial IPEH is likely similar to that of the extracranial disease, however, the difficulty associated with the removal of some intracranial lesions complicates therapeutic options.[14]

At surgery, IPEH appears as a reddish well-demarcated mass that may bleed vigorously when manipulated. Complete surgical resection is the optimal treatment,asthe lesion can recur, sometimes rapidly and aggressively, following incomplete excision.[3, 14, 20, 24] Lesions involving the critical structures of the skull base may not be amenable to complete resection without significant morbidity. In these instances, as was true in our case, residual disease is left behind. The treatment of residual and recurrent disease has been the subject of multiple investigations. Observation with close followup is a reasonable initial option and there are cases in the literature demonstrating clinically silent residual disease without further growth over multiple years.[14, 20, 24] These cases support the avoidance of initial radical resection at the cost of irreversible neurologic deficit.

Symptomatic or rapidly recurring disease has been treated with re-resection, radiosurgery, and/or chemotherapy. Kristof et al. treated a three-month postoperative recurrence of cavernous sinus and sellar IPEH with local irradiation.[14] There was sustained shrinkage of the recurrence at 3.5 years. Avellino et al. reported on a subtotallyresected cerebellopontine angle IPEH in which residual disease was treated with postoperative radiation for a total 45-Gy dose.[3] Follow-up at 18 months revealed regression of the residual mass and imaging at three years revealed stable disease. Nine years after the initial surgery, the patient became increasingly symptomatic and MRI demonstrated a large recurrence extending into the middle cranial fossa. Aggressive surgical resection achieved a neargross-total resection, however, recurrence was again found on 15-month follow-up MRI. The recurrence was treated with gamma knife radiosurgery (GKR), resulting in stabilization of the recurrent disease at 2.75 years. Ohshima et al. demonstrated a reduction in lesion size and improvement in symptoms with GKR at two-year follow-up for residual IPEH of the middle cranial fossa.[21]Chemotherapy has been recommended by some authors, however, there is no sufficient evidence currently available to confirm benefit.[8]

Our case is unique due to the co-occurrence of a prolactinoma and incidentalcavernous sinus IPEH.Despite the close proximity between the lesions, they represent two distinct and unrelated pathologies. The IPEH, although large and completely filling the cavernous sinus, was completely asymptomatic at the time of presentation. Its proximity to the symptomatic lesion, its demonstration of growth, and the need for tissue diagnosis made simultaneous surgical resection of both lesions the 
optimal treatment. The accelerated rate with which significant recurrence of the IPEH occurred indicated an aggressive process requiring further treatment. Management was complicated by the fact that the patient remained completely asymptomatic from the growing lesion. Radical surgical resection would undoubtedly be associated with an unacceptable risk of morbidity. Although the literature is relatively sparse, GKR has been shown to achieve good control of IPEH recurrences in multiple cases. Close radiographic follow-up in this patient will be required to determine if the lesion responds to radiosurgery. Future investigations into the underlying pathophysiology of IPEH are required to better detail adjuvant treatment protocols for lesions not amenable to complete surgical resection.

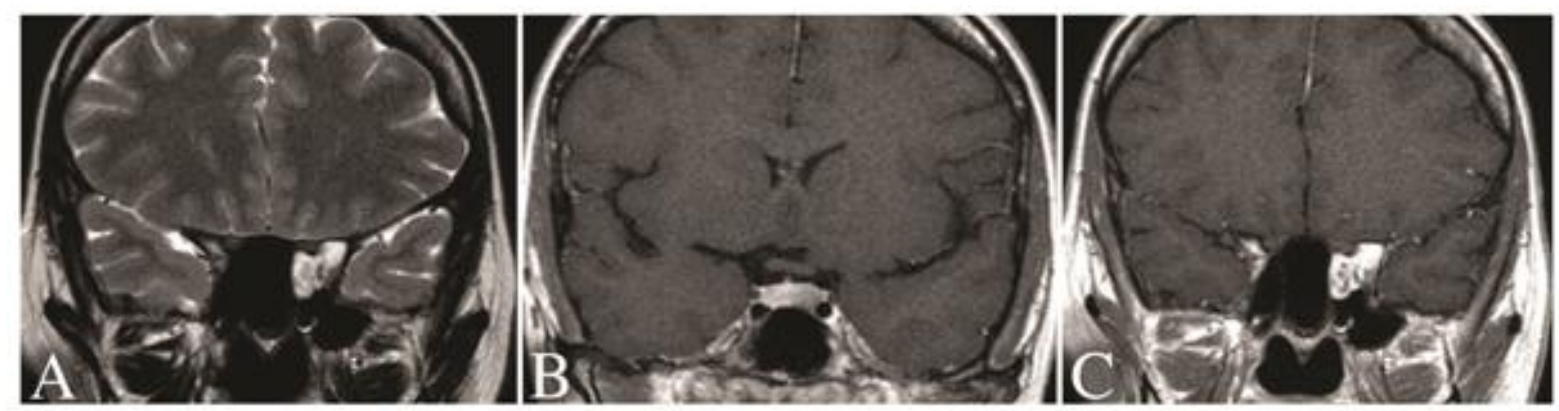

Figure1. Preoperative MRI. (A) Coronal T2-weighted MRI demonstrating a 2x1.4x1.7 cm hyperintense mass within the left cavernous sinus. (B) Coronal T1-weightedMRI with gadolinium demonstrating an $8 \times 8 \times 8 \mathrm{~mm}$ leftsided sellarnonenhancingmicroadenoma. (C) An additional slice through the coronal T1-weighted MRI with gadolinium reveals the homogenously enhancing left cavernous sinus mass.
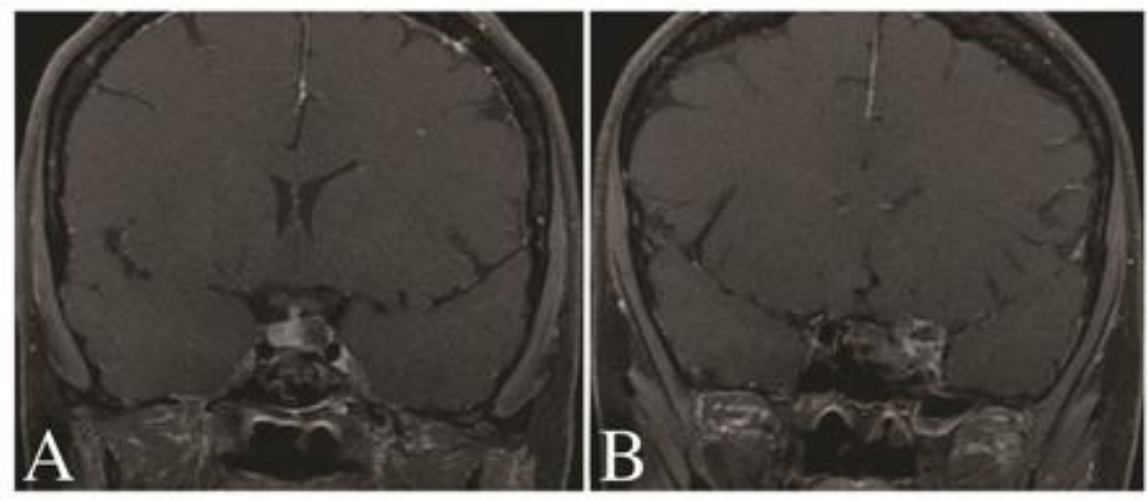

Figure2. Postoperative MRI. (A) Postoperative coronal Tl-weighted MRI with gadolinium demonstrates complete resection of the pituitary microadenoma. (B) Residual enhancement is observed in the left cavernous sinus, which is consistent with residual IPEH.

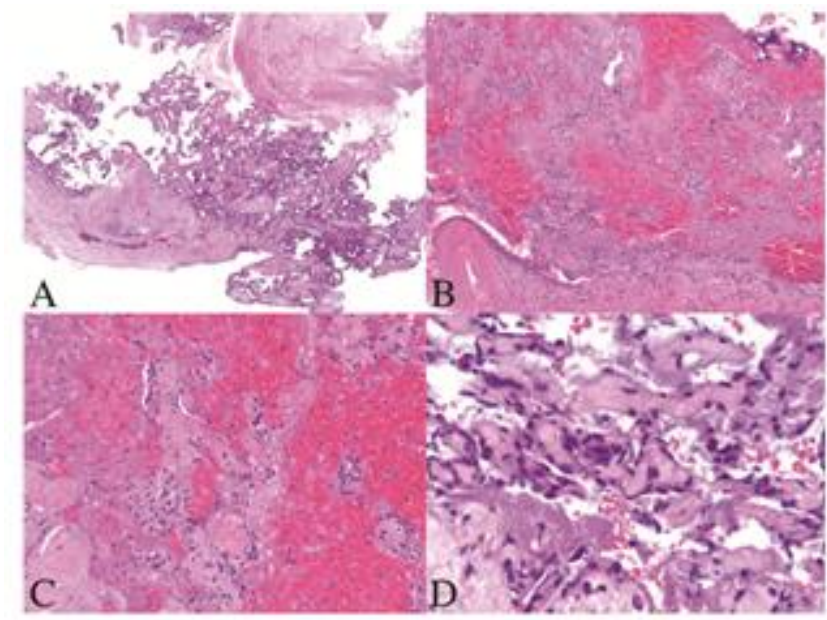

Figure3. Histology of permanent specimens. A) Multiple small papillary structures are evident at low power magnification. $B$ and $C$ ) Fresh blood and fibrin are seen admixed with the endothelialized hyaline papillary cores. D) The core of the papillary structures is composed of acellularhyalinizedfibrocollagenous tissue. They are lined by endothelial cells with little or no atypia; they are immunoreactive to immunohistochemistry for CD31. Mitotic activity is not present. 


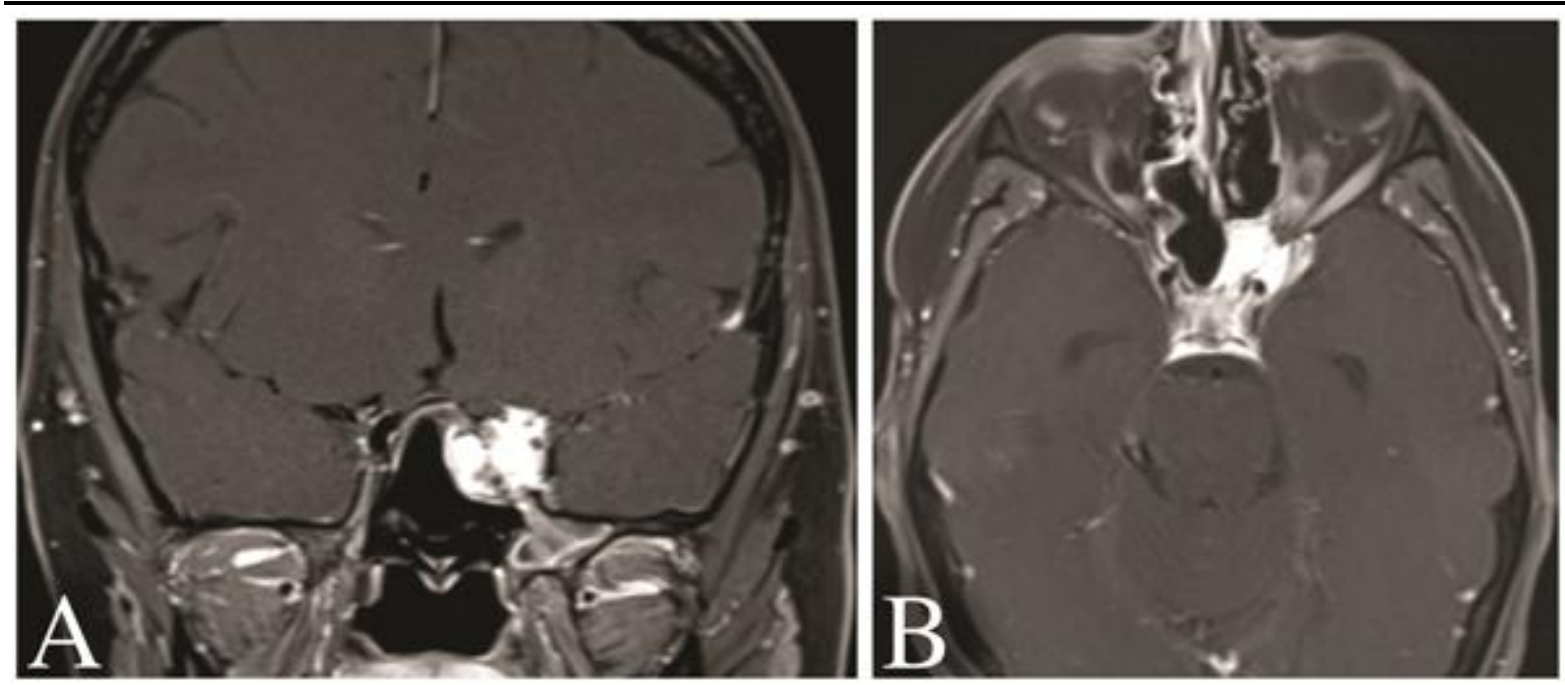

Figure4. MRI at six-month follow-up. Six-month postoperative coronal (A) and axial (B) T1-weighted MRI with gadolinium demonstrates $2.2 \times 1.5 \times 1.9 \mathrm{~cm}$ enhancing mass in the left cavernous sinus consistent with IPEH recurrence.

\section{REFERENCES}

[1] Abdel-Majid RM, Marshall JS. Prostaglandin E2 induces degranulation-independent production of vascular endothelial growth factor by human mast cells. Journal of immunology 2004;172(2):1227-36.

[2] Albrecht S, Kahn HJ. Immunohistochemistry of intravascular papillary endothelial hyperplasia. Journal of cutaneous pathology 1990;17(1):16-21.

[3] Avellino AM, Grant GA, Harris AB, Wallace SK, Shaw CM. Recurrent intracranial Masson's vegetant intravascular hemangioendothelioma. Case report and review of the literature. Journal of neurosurgery 1999;91(2):308-12.

[4] Barbera-Guillem E, Nyhus JK, Wolford CC, Friece CR, Sampsel JW. Vascular endothelial growth factor secretion by tumor-infiltrating macrophages essentially supports tumor angiogenesis, and $\mathrm{IgG}$ immune complexes potentiate the process. Cancer research 2002;62(23):7042-9.

[5] Barr RJ, Graham JH, Sherwin LA. Intravascular papillary endothelial hyperplasia. A benign lesion mimicking angiosarcoma. Archives of dermatology 1978;114(5):723-6.

[6] Buchner A, Merrell PW, Carpenter WM, Leider AS. Oral intravascular papillary endothelial hyperplasia. Journal of oral pathology \& medicine : official publication of the International Association of Oral Pathologists and the American Academy of Oral Pathology 1990;19(9):41922.

[7] Cagli S, Oktar N, Dalbasti T, Islekel S, Demirtas E, Ozdamar N. Intravascular papillary endothelial hyperplasia of the central nervous system--four case reports. Neurologia medicochirurgica 2004;44(6):302-10.

[8] Crocker M, deSouza R, Epaliyanage P, Bodi I, Deasy N, Selway R. Masson's tumour in the right parietal lobe after stereotactic radiosurgery for cerebellar AVM: case report and review. Clinical neurology and neurosurgery 2007;109(9):811-5.

[9] Garcia-Macias MC, Abad M, Alonso MJ, Flores T, Bullon A. Masson's vegetant intravascular hemangioendothelioma. Fine needle aspiration cytology, histology and immunohistochemistry of a case. Acta cytologica 1990;34(2):175-8.

[10] Hagiwara A, Inoue Y, Shakudo M, Wakasa K, Sato K, Ohata K, Daikokuya H, Yamada R. Intracranial papillary endothelial hyperplasia: occurrence of a case after surgery and radiosurgery. Journal of computer assisted tomography 1999;23(5):781-5.

[11] Hashimoto H, Daimaru Y, Enjoji M. Intravascular papillary endothelial hyperplasia. A clinicopathologic study of 91 cases. The American Journal of dermatopathology 1983;5(6):53946. 
[12] Iijima K, Yoshikawa N, Nakamura H. Activation-induced expression of vascular permeability factor by human peripheral T cells: a non-radioisotopic semiquantitative reverse transcriptionpolymerase chain reaction assay. Journal of immunological methods 1996;196(2):199-209.

[13] Kim S, Jun JH, Kim J, Kim do W, Jang YH, Lee WJ, Chung HY, Lee SJ. HIF-1alpha and VEGF expression correlates with thrombus remodeling in cases of intravascular papillary endothelial hyperplasia. International journal of clinical and experimental pathology 2013;6(12):2912-8.

[14] Kristof RA, Van Roost D, Wolf HK, Schramm J. Intravascular papillary endothelial hyperplasia of the sellar region. Report of three cases and review of the literature. Journal of neurosurgery 1997;86(3):558-63.

[15] Lee W, Hui F, Sitoh YY. Intravascular papillary endothelial hyperplasia in an intracranial thrombosed aneurysm: 3T magnetic resonance imaging and angiographical features. Singapore medical journal 2004;45(7):330-3.

[16] Makos C, Nikolaidou A. Intravascular papillary endothelial hyperplasia (Masson's tumor) of the oral mucosa. Presentation of two cases and review. Oral Oncology 2004 40:59-62.

[17] Masson MP. L'Hemangioendotheliome intravasculaire. Ann Anat Pathol 1923;93:517.

[18] Miller TR, Mohan S, Tondon R, Montone KT, Palmer JN, Zager EL, Loevner LA. Intravascular papillary endothelial hyperplasia of the skull base and intracranial compartment. Clinical neurology and neurosurgery 2013;115(10):2264-7.

[19] Moon WS, Chung GH, Hong KH. Intravascular papillary endothelial hyperplasia in a vascular lesion of the paranasal sinus. Archives of pathology \& laboratory medicine 2000;124(8):1224-7.

[20] Nagib MG, Sung JH, Seljeskog EL. Neurocutaneous Masson's vegetant intravascular hemangioendothelioma. Neurosurgery 1982;11(6):800-3.

[21] Ohshima T, Ogura K, Nakayashiki N, Tachibana E. Intravascular papillary endothelial hyperplasia at the superior orbital fissure: report of a case successfully treated with gamma knife radiosurgery. Surgical neurology 2005;64(3):266-9; discussion 9.

[22] Patt S, Kaden B, Stoltenburg-Didinger G. Intravascular papillary endothelial hyperplasia at the fissura orbitalis superior: a case report. Clinical neuropathology 1992;11(3):128-30.

[23] Shih CS, Burgett R, Bonnin J, Boaz J, Ho CY. Intracranial Masson tumor: case report and literature review. Journal of neuro-oncology 2012;108(1):211-7.

[24] Sickler GK, Langford LA. Intracranial tumor-forming papillary endothelial hyperplasia--a case report. Clinical neuropathology 1990;9(3):125-8.

[25] Wehbe MA, Otto NR. Intravascular papillary endothelial hyperplasia in the hand. The Journal of hand surgery 1986;11(2):275-9. 